\title{
Fiber optic index sensor enhanced by gold nanoparticle assembly on long period grating
}

\author{
Fei $\operatorname{Tian}^{1}{ }^{1}$, Xiangzhi $\mathrm{Li}^{1}$, Jiri Kanka ${ }^{2}$ and Henry $\mathrm{Du}^{1}$ \\ ${ }^{1}$ Department of Chemical Engineering and Materials Science, Stevens Institute of Technology, Hoboken, New Jersey 07030 \\ ${ }^{2}$ Institute of Photonics and Electronics AS CR, v.v.i., Chaberska 57, Prague 8, 18251 Czech Republic \\ *Corresponding author: ftian1@stevens.edu
}

Received Month X, XXXX; accepted Month X, XXXX; posted online Month X, XXXX

\begin{abstract}
The sensing performance of long period grating (LPG) in integration with colloidal gold nanoparticles (LPG-AuNPs) is investigated. An LPG coupled with a higher cladding mode of $\mathrm{LP}_{0,10}$ is fabricated on single mode fiber (SMF) with higher sensitivity to refractive index compared with lower cladding modes such as $\mathrm{LP}_{0,2}, \mathrm{LP}_{0,3}$ and $\mathrm{LP}_{0,4}$ via symmetric $\mathrm{CO}_{2}$ laser irradiation. A bilayer of poly (allylamine hydrochloride) (PAH) and gold nanoparticles is deposited on the grating portion via layer-by-layer self-assembly technique. It is found that the sensitivity is increased from $460 \mathrm{~nm} / \mathrm{refractive}$ index unit (RIU) to $759 \mathrm{~nm} / \mathrm{RIU}$ using the LPG-AuNPs.
\end{abstract}

Key words: Long period grating, sensor, Au nanoparticle, layer-by-layer

Optics techniques have been considered as a powerful tool for many sensing applications [1-5]. Particular attention has been focused on the optic fiber long period grating (LPG) based sensor owing to their advantages such as low insertion loss, electromagnetic field immunity, simple fabrication, low back reflection and fast speed. For the above reasons the LPG has been used for a wide range of applications in both optic fiber communication applications such as band-rejection filters [6], gain equalizers [7], and dispersion compensation [8], as well as in optic sensing systems such as gas, chemical and biological sensors [9-15]. The resonance condition for light coupled from the fundamental core mode to the $\mathrm{co}^{-}$ propagating cladding mode is governed by the phase matching condition $\lambda_{F}=\left(n^{\mathrm{eff}}\right.$, core ${ }^{-} n^{\mathrm{eff}}$, $\left.\operatorname{clad}(\hat{)})\right) \Lambda$, where $\lambda_{i}$ is the resonance wavelength of the $t$ th cladding mode, and $n^{\mathrm{eff}}$, core and $n^{\mathrm{eff}}$ clad $(\lambda)$ are the effective refractive indices of the fundamental core mode and the ith cladding mode, respectively. The mode coupling results in a series of attenuation bands in the transmission spectrum of the LPG. A part of the power distribution of the cladding mode extends to the surrounding of the cladding as the evanescent field, thus the cladding attenuation is dependent on the optical properties of the surrounding media. This makes the LPG an excellent candidate for refractive index (RI) and chemical sensing. By careful design, the LPG can be extremely sensitive to the RI of the surrounding media. For example, recent study shows that the higher the cladding mode, the higher the sensitivity is to the deposition of the thin film in the portion of grating for an LPG fabricated in SMF-28 [16]. At the same time, the integration of optical fiber and nanomaterials such as gold or silver nanoparticles has drawn a lot of interest due to their foreseen prospect in high sensitivity, special functionality and specific response, resulting in the potential applications in chemical and biological sensing [17-19]. Sergiy Korposh et al. demonstrate the ability of LPG modified with a film nanoassembled by the alternate deposition of $\mathrm{SiO}_{2}$ nanoparticles and poly(diallyldimethyl ammonium chloride) (PDDA) to obtain quantitative measurements for the detection of organic compounds that can be infused into the porous film, which changes the RI of the film [20]. Another study demonstrated an improved RI sensitivity from $-17.93 \mathrm{~nm} /$ refractive index unit (RIU) to $-23.45 \mathrm{~nm} /$ RIU by incorporating a layer of colloidal gold nanoparticles (AuNPs) on the LPG fabricated in SMF-28 [21]. There are several approaches for the deposition of thin films of sub-um thickness onto the portion of LPG, such as electrostatic self-assembly (ESA) [18], LangmuirBlodgett (LB) [17], layer-by-layer (LbL) self-assembly [16] and dip-coating [22, 23] techniques. Several research groups have demonstrated optical sensors for sensitive and selective measurements for $\mathrm{pH}$, humidity, and chemical and biological components [24-31] though surface modification of the LPG.

In this study we explored the enhancement of the sensing performance of LPG with AuNPs. Since highorder cladding modes such as LP 0,10 in LPG are significantly more sensitive than their lower-order counterparts [16], $\mathrm{LP}_{0,10}$ is coupled in the LPG during the fabrication process in order to achieve a high RI sensitivity. $\mathrm{Au}$ NPs were prepared by adding $4 \mathrm{~mL} 1 \%$

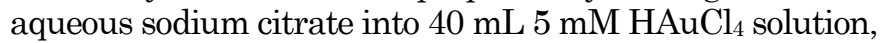
which was exposed to UV lamp for 30 min under stirring [32]. A bilayer of polyelectrolyte poly(allylamine hydrochloride) and AuNPs with the average diameter of $\sim 50 \mathrm{~nm}$ is deposited on the LPG through the LbL assembly technique. The $\mathrm{LbL}$ process is an efficient way for the deposition of functional coatings on the LPG in that it is easy to control the coverage density and thickness of the coating by changing the deposition conditions such as $\mathrm{pH}$ of the polyelectrolyte solution, concentrations and layer number. The LPGs with and without the AuNPs are used for the RI sensitivity test. It has shown that the LPG with AuNPs has a sensitivity of $759 \mathrm{~nm} / \mathrm{RIU}$, whereas the LPG without AuNPs has a RI sensitivity of $460 \mathrm{~nm} / \mathrm{RIU}$. 
$\mathrm{LP}_{0,10}$ cladding mode is coupled in the conventional optical fiber SMF-28 using symmetric point-by-point $\mathrm{CO}_{2}$ laser irradiation. Details of the setup of the fabrication can be found in refs [33, 34]. In order to compare the difference in sensitivity with and without AuNPs, two LPGs are fabricated with the same parameter. The LPGs have a period of $240 \mu \mathrm{m}$ and a length of $37 \mathrm{~mm}$. AuNPs are deposited onto one of the LPGs via LbL technique. Basically, a layer of poly (allylamine hydrochloride) (PAH) with a concentration of $0.2 \mathrm{mg} / \mathrm{mL}$ and $\mathrm{pH}$ of 7.0 is deposited for 20 minutes as the first layer on the LPG. The PAH is a positively charged polymer in solution. And then negatively charged AuNPs with an average diameter of $50 \mathrm{~nm}$ is deposited at $\mathrm{pH} 7.0$ for 80 minutes as a second layer on the LPG through electrostatic interaction with the previous PAH layer. Fig. 1 shows the scanning electron microscope image of the AuNPs on the LPG. Fig. 2 shows the transmission spectra of the LPG before and after the deposition of the PAH/AuNPs coating.

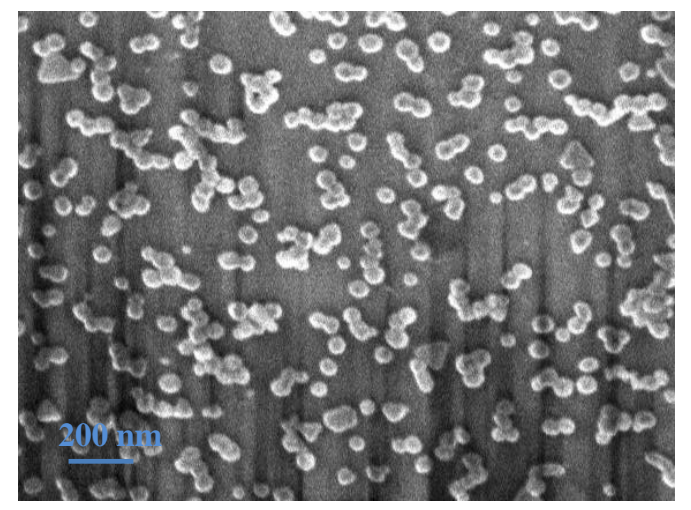

Fig. 1. (Color online) SEM image of the AuNPs deposited on the LPG.

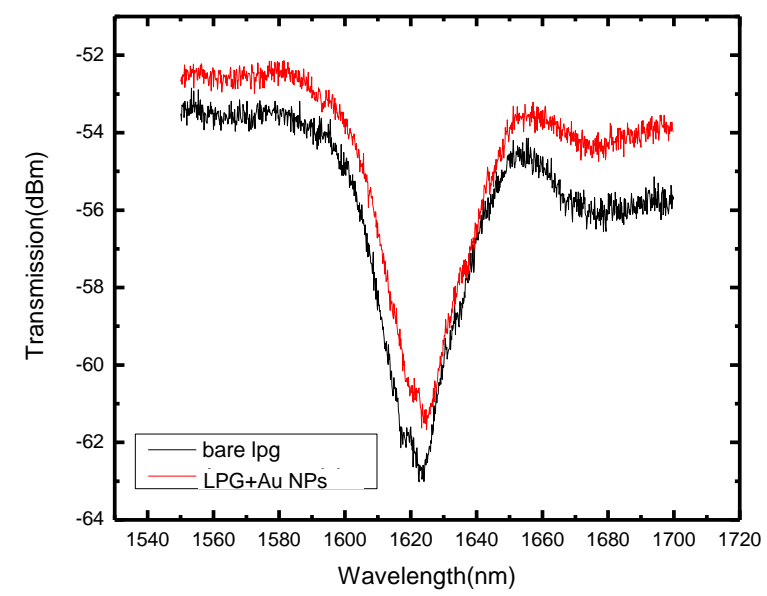

Fig. 2. (Color online) Transmission spectra of the LPG before and after the deposition of PAH/AuNPs.

From Fig. 2 we see that the resonance wavelength of the LPG has a red shift when there is a PAH/AuNPs coating deposited. The deposition of the coating changes the effective indices of the core and cladding mode of the $\mathrm{LPG}$ so that $\partial \mathrm{n}^{\text {neff }}$ core $(\lambda \mathrm{i}, \quad \mathrm{nsur}) / \partial \mathrm{n}_{\text {sur }}>\partial \mathrm{n}^{\text {neff }}$ clad(i) $(\lambda \mathrm{i}$, $\mathrm{nsur}) / \partial \mathrm{n}_{\text {sur, }}$ resulting in an increase in the resonance wavelength and thus a red shift. Here $\mathrm{n}_{\text {sur }}$ is the RI of the surrounding medium. After the deposition of the PAH/AuNPs on the LPG, its response to the surrounding $\mathrm{RI}$ is measured using air, water, ethanol and $40 \mathrm{wt} \%$ glycerin/water solution as means to alter the RI of the surrounding medium, the refractive indices of which are 1 , $1.333,1.361$ and 1.384 , respectively. At the meantime, the LPG without the AuNPs is also immersed in the same series of surrounding media to measure its RI sensitivity. The response of the LPG with and without the PAH/AuNPs coating are shown in Fig. 3.
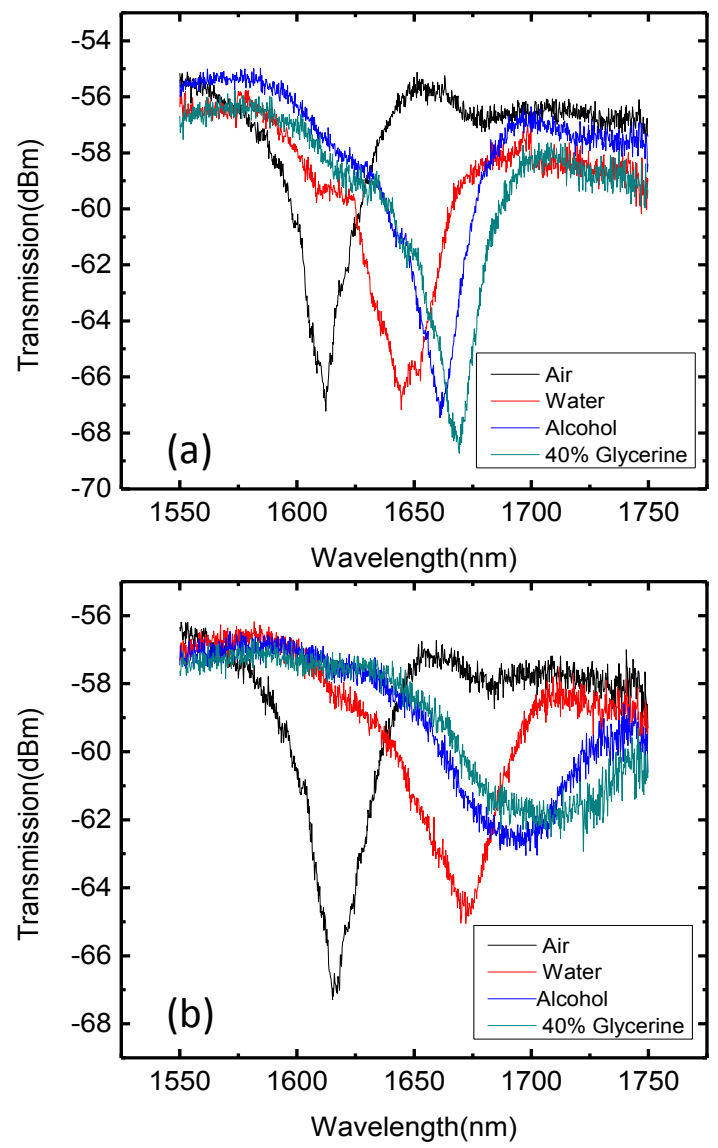

Fig. 3. (Color online) Transmission spectra of the response of LPGs (a) without and (b) with PAH/AuNPs coating when immersed in air, water, ethanol and $40 \mathrm{wt} \%$ glycerin-water mixture, respectively.

Fig. 3 shows that for both LPGs, there is a red shift in the resonance wavelength when the RI of the surrounding medium increases from that of air to that of the glycerinwater mixture. However, the amounts of the red shift with respect to the RI change are different. Interestingly, for the LPG with the Au NPs, not only the resonance wavelength shifts more rapidly, but also the attenuation band becomes shallower when the surrounding RI is greater than that of water.

In order to calculate the RI sensitivity of the two LPGs, the resonance wavelengths are plotted as a function of the $\mathrm{RI}$ of the surrounding medium in Fig. 4. Fig. 4 shows that the response of the LPG to the surrounding medium is not 
linear throughout all the $\mathrm{RI}$ range. But when it is measured in liquid phase, the response becomes linear. We take the response in the liquid phase and fit it with a linear equation in order to calculate the RI sensitivities of the two LPGs and do the comparison. Fig. 5 shows the linear fitting for the two LPGs measured using water, ethanol and glycerin-water mixture.

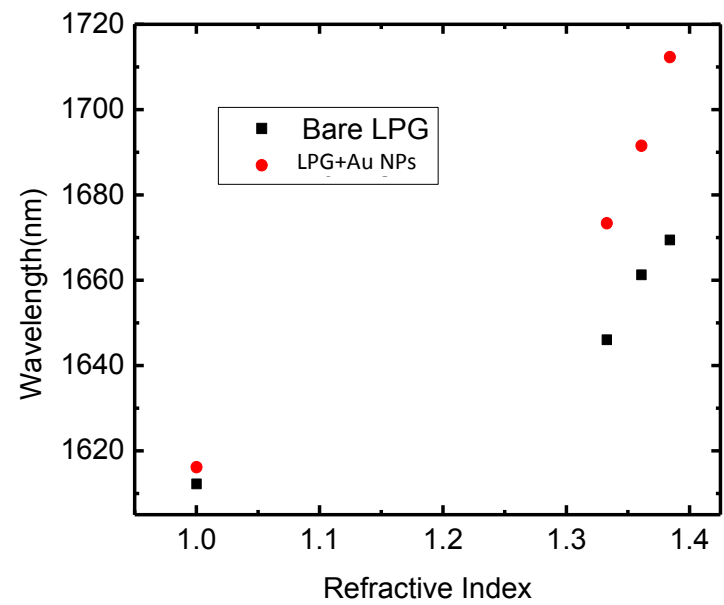

Fig. 4. (Color online) Response of LPGs with and without $\mathrm{PAH} / \mathrm{AuNPs}$ coating to the surrounding RI.

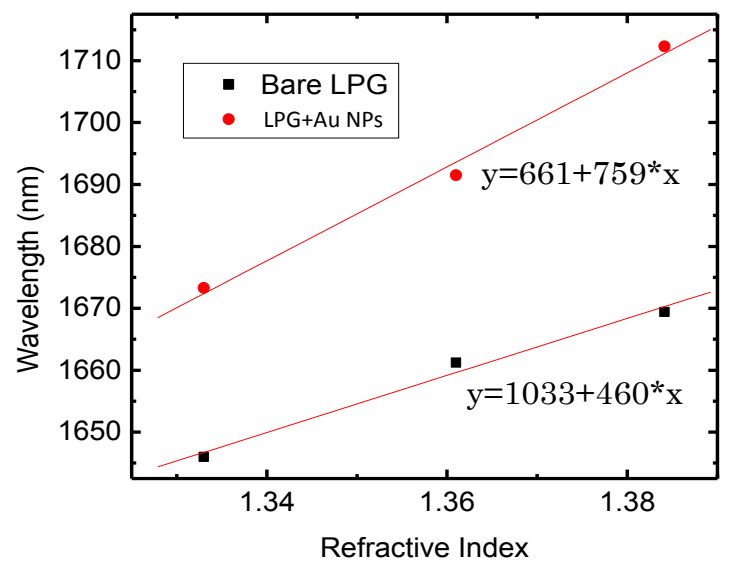

Fig. 5. (Color online) Linear fitting of the response of LPGs with and without PAH/AuNPs coating to the surrounding RI.

The slope for each linear fitting represents the RI sensitivity of each LPG. Fig. 5 shows that in the linear region, the LPG with AuNPs has a higher RI sensitivity than the LPG without AuNPs. The LPG with the AuNPs coating has a RI sensitivity of $759 \mathrm{~nm} / \mathrm{RIU}$, while the LPG without AuNPs coating has a RI sensitivity of 460 $\mathrm{nm} / \mathrm{RIU}$. The sensitivity is improved by $65 \%$ through the incorporation of the AuNPs with the LPG. The AuNPs have a large surface area which tends to increase the total surface for the analyte during the sensing interrogation. $\mathrm{Au}$ surface is unique in many ways for rich and versatile chemical and biological functionalization hence its incorporation as part of the LPG broadens the opportunity for sensing and process monitoring such as label-free detection and biomolecular binding at the surface of AuNPs.

In conclusion, we have investigated the RI sensitivity of SMF-LPG integrated with AuNPs via LbL self-assembly. RI measurement shows that the sensitivity of the LPG can be increase from $460 \mathrm{~nm} / \mathrm{RIU}$ to $759 \mathrm{~nm} / \mathrm{RIU}$ by measuring the response of the SMF-LPG to water, ethanol and glycerin-water mixture in the surrounding of the LPG. Although a number of studies have investigated in the chemical sensing based on LPGs integrated with thin film, the LbL approach reported here allows for a more accurate control on the deposition process of the thin film coating. Through optimization of the deposition parameters such as the coverage density and coating thickness, the sensitivity of the LPG can be further enhanced. It has the potential to be integrated with functional nano-materials for chemical and biological sensing strategies.

This work is supported by the US National Science Foundation under grant number ECCS- 1611155 and by the Ministry of Education, Youth and Sport of the Czech Republic under grant number LH 11038 within the U.S.Czech S\&T Cooperation agreement.

\section{References}

1. S. W. James, and R. P. Tatam, "Fiber optic sensors with nano-structured coatings,” J. Opt. A, Pure Appl. Opt. 8 (7), S430 (2006).

2. H. Chen, F. Tian, J. Chi, and H. Du, "Sapphire fiber opticbased surface-enhanced Raman scattering by direct and evanescent-field excitation," Proc. SPIE 9098, 90980T (2014).

3. H. Chen, F. Tian, J. Kanka, and H. Du, "A scalable pathway to nanostructured sapphire optical fiber for evanescent-field sensing and beyond,” Appl. Phys. Lett. 106, 111102 (2015).

4. H. Chen, F. Tian, J. Chi, J. Kanka, and H. Du, "Advantage of multi-mode sapphire optical fiber for evanescent-field SERS sensing," Opt. Lett. 39, 5822 (2014).

5. A. Cusano, M. Consales, A. Crescitelli, and A. Ricciardi, LabOn-Fiber Technology (Springer, 2015).

6. A. M. Vengsarkar, P. J. Lemaire, J. B. Judkins, V. Bhatina, T. Erdogan, and J. E. Sipe, "Long period fiber gratings as band-rejection filters," IEEE J. Lightwave Technol. 1996, 14, 58-65.

7. A. M. Vengsakar, J. R. Pedrazzani, J. B. Judikins, N. S. Lemaire, N. S. Bergano, and C. R. Davidson, "Long-period fiber-grating based gain equalizers," Opt. Lett. 1996, 21, 336-338.

8. M. Das, and K. Thyagarajan, "Dispersion compensation in transmission using uniform long period fiber gratings," Opt. Commun. 2001, 197, 159-163.

9. F. Tian, J. Kanka, X. Li, and H. Du, "Exploration of higherorder mode coupling in long-period gratings for sensitive monitoring of polyelectrolyte self-assembly at nanoscale," Proc. SPIE 9098, 90980R (2014).

10. Alain Trouillet, Emmanuel Marin and Colette Veillas, "Fibre gratings for hydrogen sensing," Meas. Sci. Technol. 17, 1124-1128 (2006).

11. F. Tian, J. Kanka, S. A. Sukhishvili, and H. Du, "Photonic crystal fiber for layer-by-layer assembly and measurements of polyelectrolyte thin films," Opt. Lett. 37, 4299 (2012). 
12. F. Tian, J. Kanka, and H. Du, "Long-period grating and its cascaded counterpart in photonic crystal fiber for gas phase measurement," Opt. Express 20, 20951 (2012).

13. Jian Zhang, Xiling Tang, Junhang Dong, Tao Wei, and Hai Xiao, "Zeolite Thin Film-Coated Long Period Fiber Grating Sensor for Measuring Trace Chemical," Opt. Express 16, 8317-8323 (2008).

14. F. Tian, Z. He, and H. Du, "Numerical and experimental investigation of long-period gratings in photonic crystal fiber for refractive index sensing of gas media," Opt. Lett. 37, 380 (2012).

15. Z. He, F. Tian, Y. Zhu, N. Lavlinskaia, and H. Du, "Long period gratings in photonic crystal fiber as an optofluidic label-free biosensor," Biosens. Bioelectron. 26, 4774 (2011).

16. F. Tian, J. Kanka, X. Li, and H. Du, "Monitoring layer-by" layer assembly of polyelectrolyte multi-layers using highorder cladding mode in long-period fiber gratings," Sens. Actuators B 196, 475 (2014).

17. N. D. Rees, S. W. James, R. P. Tatam, and G. J. Ashwell, "Optical fiber long-period gratings with Langmuir - Blodgett thin-film overlays," Opt. Lett. 27(9), 686-688 (2002).

18. I. Del Villar, M. Achaerandio, I. R. Matías, and F. J. Arregui, "Deposition of overlays by electrostatic selfassembly in longperiod fiber gratings," Opt. Lett. 30(7), 720-722 (2005).

19. I. Del Villar, I. R. Matías, and F. J. Arregui, "Influence on cladding mode distribution of overlay deposition on long period fiber gratings," J. Opt. Soc. Am. A 23(3), 651-658 (2006).

20. Sergiy Korposh, Stephen W. James, Seung-Woo Lee, Stephen Topliss, Sammy C. Cheung, William J. Batty, and Ralph P. Tatam, "Fiber optic long period grating sensors with a nanoassembled mesoporous film of $\mathrm{SiO}_{2}$ nanoparticles," Opt. Express 18, (2010).

21. J. -L. Tang, S. -F. Cheng, W. -T. Hsu, T. -Y. Chiang, L. -K. Chau, "Fiber-optic biochemical sensing with a colloidal goldmodified long period fiber grating," Sensors and Actuators B 119, 105-109 (2006).

22. Z. Gu, and Y. Xu, "Design optimization of a long-period fiber grating with sol-gel coating for a gas sensor," Meas. Sci. Technol. 18(11), 3530-3536 (2007).

23. A. Cusano, P. Pilla, L. Contessa, A. Iadicicco, S. Campopiano, A. Cutolo, M. Giordano, and G. Guerra, "High sensitivity optical chemosensor based on coated long-period gratings for sub-ppm chemical detection in water," Appl. Phys. Lett. 87(23), 234105 (2005).

24. S. C. Cheung, S. M. Topliss, S. W. James, and R. P. Tatam, "Response of fiber optic long period gratings operating near the phase matching turning point to the deposition of nanostructured coatings," J. Opt. Soc. Am. B 25(6), 897-902 (2008).

25. J. M. Corres, I. R. Matías, I. del Villar, and F. J. Arregui, "Design of $\mathrm{pH}$ sensors in long-period fiber gratings using polymeric nanocoatings," IEEE Sens. J. 7(3), 455-463 (2007).

26. J. Keith, L. C. Hess, W. U. Spendel, J. A. Cox, and G. E. Pacey, "The investigation of the behavior of a long period grating sensor with a copper sensitive coating fabricated by layer-by-layer electrostatic adsorption," Tatanta 70, 818-822 (2006).

27. A. Cusano, A. Iadicicco, P. Pilla, L. Contessa, S. Campopiano, A. Cutolo, M. Giordano, and G. Guerra, "Coated long-period fiber gratings as high-sensitivity opto-chemical sensors," J. Lightwave Technol. 24(4), 1776-1786 (2006).

28. S. Korposh, S. Kodaira, S.-W. Lee, W.J. Batty, S.W. James, R. P. Tatam, "Deposition of SiO2/polymer nanoporous thin films on long-period grating (LPG) optical fibers and dramatic enhancement of the resonance bands," Sensing Technology, 2008. ICST 2008, 666-669, (2008) doi: 10.1109/ICSENST.2008.4757189.

29. D. Viegas, J. Goicoechea, J. M. Corres, J. L. Santos, L. A. Ferreira, F. M. Araújo, and I. R. Matías, "A fiber optic humidity sensor based on a long-period fiber grating coated with a thin film of $\mathrm{SiO} 2$ nanospheres," Meas. Sci. Technol. 20(3), 034002 (2009).

30. Shijie Zheng, Yinian Zhu, and Sridhar Krishnaswamy, "Fiber humidity sensors with high sensitivity and selectivity based on interior nanofilm-coated photonic crystal fiber longperiod gratings," Sens. Actuators B 176, 264-274 (2013).

31. Shijie Zheng, "Long-period fiber grating moisture sensor with nano-structured coatings for structural health monitoring," Struct. Health Monit. 14, 148-157 (2015).

32. Maung Kyaw Khaing Oo, Yamin Yang, Yue Hu, Maria Gomez, Henry Du, and Hongjun Wang, "Gold NanoparticleEnhanced and Size-Dependent Generation of Reactive Oxygen Species from Protoporphyrin IX," ACS Nano 6, 1939-1947 (2012).

33. F. Tian, J. Kanka, B. Zou, K. S. Chiang, and H. Du, "Longperiod gratings inscribed in photonic crystal fiber by symmetric $\mathrm{CO}_{2}$ laser irradiation," Opt. Express 21, 13208 (2013).

34. F. Tian, J. Kanka, B. Zou, K. S. Chiang, and Henry Du, "Effect of irradiation symmetry of $\mathrm{CO} 2$ laser on mode coupling in long-period gratings inscribed in photonic crystal fiber," Proc. SPIE 8722, 87220I (2013). 